\title{
Manipulation of the quantum state by Majorana transition in spinor Bose-Einstein condensates
}

\author{
Lin Xia, Xu Xu, Fan Yang, Wei Xiong, Juntao Li, Qianli Ma, Xiaoji Zhou, Hong Guo \& and Xuzong Chen \\ School of Electronics Engineering and Computer Science, \\ Peking University, Beijing 100871, Peoples Republic of China
}

(Dated: July 7, 2021)

\begin{abstract}
Manipulation of the quantum state by the Majorana transition in spinor BEC system has been realized by altering the rotation frequency of the magnetic fleld's direction. This kind of manipulation method has no limitation on the transition speed in principle and the system is well closed, which provides a new and superior tool to manipulate quantum states. Using this methord on pulsed atom laser, multicomponent spinor atom laser is generated. We demonstrate that the experiment results are agreed with the theoretical predication.
\end{abstract}

PACS numbers: 03.75.Mn, 03.75.Kk, 32.80.Pj

Precise and sophisticated control of quantum state is a major goal in atom physics and is expected to lead to new physics and new applications, such as quantum computation. An important requirements of the manipulation process in quantum computation is to be fast (in order to minimize the effects of decoherence unavoided in real quantum system, and to speed up the computation). In principle, the Majorana transition, one kind of nonadiabatic transition, has no limitation on the transition speed, which has the potential to give ultra fast manipulation of quantum states. Another advantage of this manipulation is that the system is well closed if we choose the ground state of hyperfine structure. This avoids the decoherence from excited states, such as atoms loses and spontaneous emission.

Majorana transition was first studied in 1932 by Majorana 1], who derived the expression of the transition in two level system: $P_{1 / 2,-1 / 2}=\exp \left(-\frac{f_{L a r}}{f_{\text {Rot }}}\right)$ where $f_{\text {Lar }}$ is the Larmor rotation frequency, $f_{\text {Rot }}$ the rotation frequency of the magnetic field (RFM). After that few quantitative results on experiments [2, 3] were reported due to the limitation of experimental techniques, e.g., the huge velocity distribution of atom beams and the nonideal distribution of magnetic field in space. The recent experimental realization of Bose-Einstein condensation provides the possibility to precisely study Majorana transition since the ensemble can be seen as motionless in the trap. Some experiments did show the effects of Majorana transition, which are qualitatively observed [4, 5]. The observation of population oscillation induced by the variation of $f_{\text {Lar }}$ was also reproted in [6], although the population subjects to the position that atoms move to in the magnetic trap, which is not in a real sense of manipulation for practical applications.

In spinor Bose-Einstein condensate system, spin comprises a degree of freedom, where the order parameter is a vector rather than a scalar in common BEC system. This system offers a new kind of coherent matter with complex internal quantum structure and rich dynamics, which have been shown in the latest investigation
7, 8, 9, 10, 11, 12. As many proposals on quantum computation and entanglement based on spinor condensates are given [13, 14, 15, 16], experimental manipulation on the spinor freedom degree and investigation on dynamics of spinor condensate system become particularly important.

In this paper, we introduce how to utilize the Majorana transition to control quantum states by adjusting $f_{\text {Rot }}$, which provides a powerful tool for quantum states manipulation. The Majorana transition is precisely studied in spinor BEC system without the disturbance of atoms huge velocity distribution in early atom beam experiments [2, 3]. An analytical expression has been developed and matches the experimental data. The quantum state of atom laser is manipulated and multicomponent spinor atom laser with symmetrical population is generated by using this method on pulsed atom laser. We also show that the Bose-Einstein condensate is most suitable for studying Majorana transition because low optical density of images and overlapping of different components will occur as the temperature increases.

We get samples of condensates in a compact low power quadrupole-Ioffe-configuration (QUIC) trap with trapping frequency $\omega_{r}=2 \pi \times 220 \mathrm{~Hz}$ radially and $\omega_{z}=2 \pi \times 20$ axially. The trap consists of a pair of quadrupole coils which are assumed to be along $x$-direction, and one Ioffe coil along $z$-direction. The direction of gravity is assumed to be $y$-direction. We design $8 \mathrm{~mm}$ aperture along the axis of Ioffe coile, which provides a good optical access for experiments. The shape of coiles is frustum of a cone in order to provide a larger area to contact with the water cooled framework. The current is 20.7A in quadrupole coils and $20.5 \mathrm{~A}$ in Ioffe coil. Typically, a ${ }^{87} R b$ condensate with $2 \times 10^{5}$ atoms at $\left|F=2, m_{F}=2\right\rangle$ state is formed after evaporative cooling.

We redesign the circuit for the turn-off of coils to control the switching off time of two type coils separatively. The Ioffe coil is in parallel with a variable resistor, and the turn-off time of Ioffe coil is set by $L_{I} / R_{I}$, where $L_{I}$ is the inductance of the coil and $R_{I}$ is the resistance of 
the loop. Conveniently, the turn-off time of Ioffe coil can be tuned continuously by setting the resistance of the variable resistor. Similarly, the quadrupole coils are in parallel with another resistor. To control the switching off time of the magnetic field separately, the eddy current must be strictly removed. We redesign the magnetic trap with grooves in the framework and get rid of the influence of eddy current, which can be shown by comparing the decreasing process detected by a detective coil with by the Hall effect current sensor.

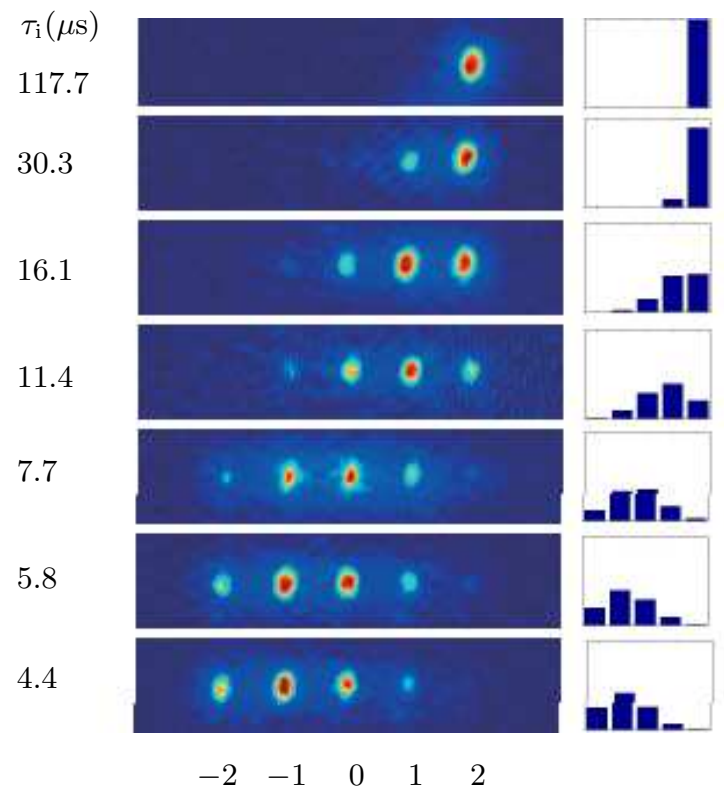

FIG. 1: Observation of different components vs the turn-off time of Ioffe coil: The atoms are initially prepared in $\mid F=2$, $\left.m_{F}=2\right\rangle$ state. The five parts of the condensates from right to left, also the same as the following figures, refer to the five states $|2,2\rangle,|2,1\rangle,|2,0\rangle,|2,-1\rangle$ and $|2,-2\rangle$ respectively. The dimension of each picture is $1.2 \mathrm{~mm} \times 0.3 \mathrm{~mm}$. During the process, the turn-off time of quadrupole coils is fixed at $\tau_{q}=$ $117.7 \mu \mathrm{s}$. We also show the population distributions obtained from the analytical expression in the bar charts.

When the nonsynchronous decreasing process of the magnetic field from different coils emerges, the condensate atoms will experience the zero field of $B_{z}=0$ and the reversion of the direction. This is easy to understand from the formation of the magnetic field. We know that the Ioffe coil's field is in the opposite direction of that of quardupole coils along $z$ direction, and the total field's direction is along the Ioffe's field. If the Ioffe's field decays fast, the zero field emerges and the field's direction reverses in $z$ direction. In this case, Majorana transition happens and the different components of BEC will be separated in space by the Stern-Gerlach effect induced by the gradient of the magnetic field. If the turn-off speed of Ioffe coil increases, the reversion speed of field increases too, which induces the increasing of $f_{\text {Rot }}$. Then more spinor components are transited from initial component $\left|F=2, m_{F}=2\right\rangle$. Setting $\tau_{i}$, the RFM $f_{\text {Rot }}$ at the time of reversion is uniquely determined as $\tau_{q}$ is a constant, where $\tau_{i}$ and $\tau_{q}$ are the $1 / e$ turn-off time of Ioffe ciol and quadrupole coils, respectively. Fig. 1 shows the relation between Majorana transition and turn-off time of the Ioffe coil in experiments. The distribution of different spinor components subjects to Majorana transition, which is controlled by adjusting the turn-off time of Ioffe coil. The distribution of spinor components well fits the theoretical prediction (see Fig. 1 right charts), which is described by Eq. (10) and (4). When $\tau_{i}=\tau_{q}$, the magnetic field does not reverse in $z$ direction and the single component condensate in $\left|F=2, m_{F}=2\right\rangle$ forms (see Fig. 1). As $\tau_{i}$ decreases, the $f_{\text {Rot }}$ increases and the induced Majorana transition becomes stronger.

To investigate the Majorana transition process in experiments more accurately, we give the theoretical analysis in the following. Majorana studied the transition in the model where the magnetic field evolves as $B_{x}(t)=0, B_{y}(t)=$ const, $B_{z}(t)=K t$ and $t=(-\infty, \infty)$. The Majorana formula has been derived both from quantum mechanics and group theory [1] 17]. For multilevel system with a total angular moment $J$,

$$
\begin{aligned}
& P_{m, m^{\prime}}=(J+m) !\left(J+m^{\prime}\right) !(J-m) !\left(J-m^{\prime}\right) !\left(\cos \frac{\theta}{2}\right)^{4 J} \\
& \times\left[\sum_{\nu=0}^{2 J} \frac{(-1)^{\nu}\left(\tan \frac{\theta}{2}\right)^{2 \nu-m+m^{\prime}}}{\nu !\left(\nu-m+m^{\prime}\right) !(J-m-\nu) !\left(J-m^{\prime}-\nu\right) !}\right]^{2},
\end{aligned}
$$

where the value of $\theta$ is given by the two level transition

$$
\sin ^{2}\left(\frac{\theta}{2}\right)=P_{1 / 2,-1 / 2} .
$$

Since the result to the system with arbitrary angular momentum $J$ can be generalized from the two level system, we emphasize on the two level case.

For a motionless system during the transition process with spin moment $s=1 / 2$ in magnetic field $B(t)$, the Schrödinger equation can be written as 6 ]

$$
i \hbar\left(\begin{array}{c}
\dot{c_{1}} \\
\dot{c_{2}}
\end{array}\right)=\frac{g \mu_{B}}{\hbar} \hat{\vec{F}} \cdot \vec{B}(t)\left(\begin{array}{c}
c_{1} \\
c_{2}
\end{array}\right),
$$

where $\hat{\vec{F}}=\hbar / 2 \hat{\vec{\sigma}}$. The magnetic field which atoms experience takes the form $\vec{B}(t)=\left[0, B_{y}(t), B_{z}(t)\right]$, where $B_{x}(t)=0$ is produced by the symmetry of the trap and $B_{y}(t)$ is produced due to the dragging down of the gravity. The experimental evolution of the magnetic field due to the discharging process of coils is exponential.

In our case, the variable $\tau_{i}$ is much less than the constant $\tau_{q}$ when Majorana transition happens obviously. By taking approximation, it is appropriate to write $\vec{B}(t)$ as

$$
\begin{aligned}
& B_{y}(t) \approx A_{y} \\
& B_{z}(t) \approx A_{z}-C_{z} t
\end{aligned}
$$


where

$$
\begin{aligned}
& A_{y}=B_{y I}+B_{y Q}, \\
& A_{z}=B_{z I}-B_{z Q}, \\
& C_{z}=B_{z I} / \tau_{i}-B_{z Q} / \tau_{q} .
\end{aligned}
$$

$B_{y I}$ and $B_{y Q}$ are the initial magnetic field respectively generated by Ioffe coil and quadrupole coils in $y$ direction, $B_{z I}$ and $B_{z Q}$ are in $z$ direction. The expressions of the RFM and Larmor frequency are $f_{\text {Rot }}=\partial B(t) / 2 \pi B(t) \partial t$ and $f_{\text {Lar }}=g \mu_{0} B(t) / 2 \pi \hbar$, respectively. In the case of Eq. (3), $f_{R o t}=C_{z} / 2 \pi A_{y}$ at the time when the magnetic field reverses its direction. From the expression of $C_{z}$, we can see that the rotation frequency of the magnetic field is only determined by $\tau_{i}$ as $\tau_{q}$ and $A_{y}$ are fixed. By substituting Eq. (3) into Eq. (2), the second-order differential equations which can be transformed into Webber equations [18] are obtained. The asymptotic solution of the Webber equation corresponding to the experiments can be derived as

$$
P_{1 / 2,-1 / 2}=\exp \left(-K \frac{A_{y}^{2}}{\frac{B_{z I I}}{\tau_{i}}-\frac{B_{z Q}}{\tau_{q}}}\right)
$$

where $K$ is a constant. The population of different spinor quantum states can be controlled by adjusting the switching off time $\tau_{i}$ in Eq. (4), which is the only adjustable parameter. This expression reveals the essence of the Majorana transition observed generally in experiments.
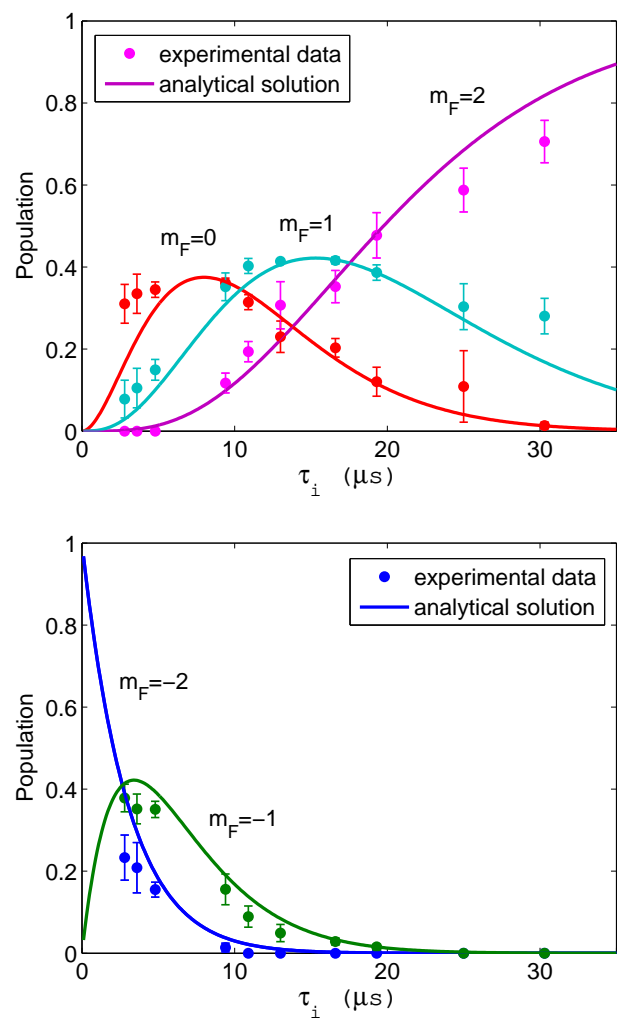

FIG. 2: Evolution of population as the turn-off time of Ioffe coil $\tau_{i}$ with atoms initially in $|F=2, m F=2\rangle$ state: The experimental data and analytical solution are shown.
The comparison between the theoretical prediction and the experimental results based on the experiments of Fig. 1 is shown in Fig. 2, which describes the evolution of spinor BEC with different turn-off time of Ioffe coile. The experimental data and theoretical results with atoms initially prepared in $\left|F=2, m_{F}=2\right\rangle$ state are shown and match each other. We can see that the population is initially prepared in $m_{F}=2$ and then transited to $m_{F}=-2$ state gradually through the $m_{F}=1,0$ and -1 state. The asymptotic state as $\tau_{i}$ decreases is $m_{F}=-2$ state. This corresponds to the total nonadiabatic process, in which the magnetic field reverses instantaneously so that the magnetic moment maintains its direction. The final distributation of atoms initially prepared in any spinor state can be easily derived by using Eq. (10) and (4). Similarly to optical pumping and Rabi oscillation, the Majorana transition is another choice to manipulate the quantum state transition by controlling the turn-off time in experiments.

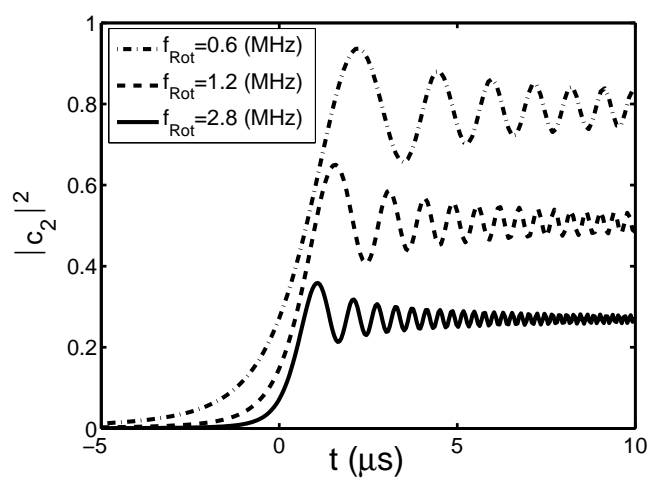

FIG. 3: Evolution of population as time in Majorana transition: Plotted is the evolution of $\left|c_{2}\right|^{2}$ as time for the three values of magnetic field rotation frequency $f_{R o t}=0.6 \mathrm{MHz}$, $1.2 \mathrm{MHz}$ and $2.8 \mathrm{MHz}$.

Fig. 3 shows the time evolution of the population by numerically solving the Webber equations. In [6], the transition period is estimated to be only about $1 \mu s$ by comparing the Larmor frequency with the field rotation frequency. Fig. 3 is another and direct way to show the transition period, which is consistent with the estimation in [6]. So the transition period length can be approximately estimated as the period length of $f_{R o t} \geq f_{L a r}$, whose expression is

$$
\Delta t=2 \cdot \sqrt{\left(\frac{B_{y} \hbar}{g \mu_{B}}\right)^{2 / 3} C_{z}^{-4 / 3}-B_{y}^{2} C_{z}^{-2}} .
$$

In the expression of $\triangle t$, the transition period is monotone decreasing and the asymptotic value is 0 (no limitation on the transition speed) at the region where $C_{z}$ tends to infinite. This trend also appears in Fig. 3.

The generation of multicomponent spinor atom laser by Majorana transition is shown in Fig. 4. We obtain atoms prepared in $m_{F}=0$ spin state by producing pulsed 


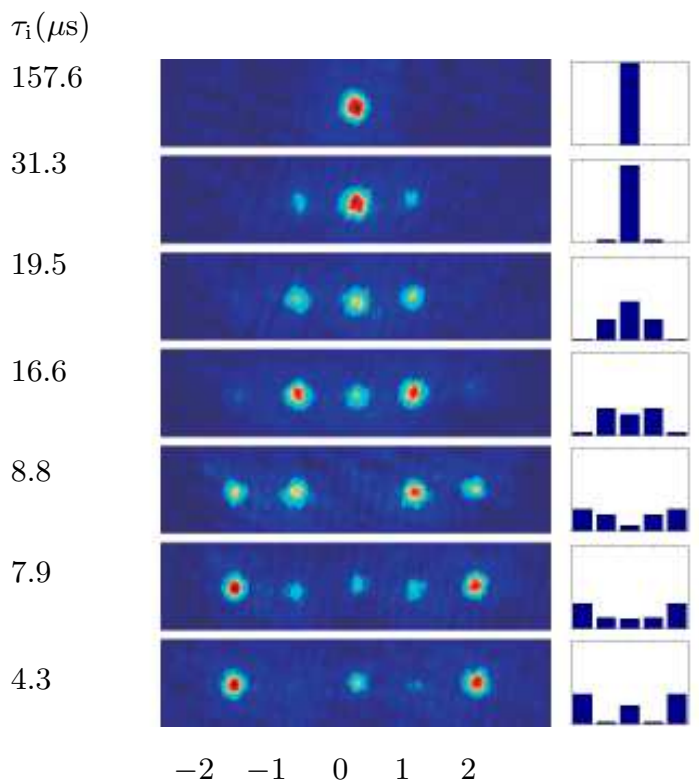

FIG. 4: Generation of multicomponent spinor atom laser by Majorana transition: The $m_{F}=0$ spinor state is prepared by producing pulsed atom laser with rf radiation. The transition process is identical to that in Fig. 1. The population distributions obtained from the analytical expression are shown in the bar charts.

atom laser with short rf radiation. The total number of atoms prepared in $m_{F}=0$ state are about $4 \times 10^{4}$. After the $m_{F}=0$ atom laser is generated and falls down for $2 \mathrm{~ms}$, the nonsynchronous process of the magnetic field happens and the multicomponent spinor atom laser is generated. We can manipulate the distribution of the population by adjusting $\tau_{i}$, which is the same as atoms initially prepared in $m_{F}=2$ state. Fig. 4 shows the symmetry of the population distrubition. This agrees with Eq. (11) when atoms are initially prepared at $m_{F}=0$ state. The generation of multicomponent spinor atom laser indicates that Majorana transition is a powerful tool for quantum state manipulation.

(a)

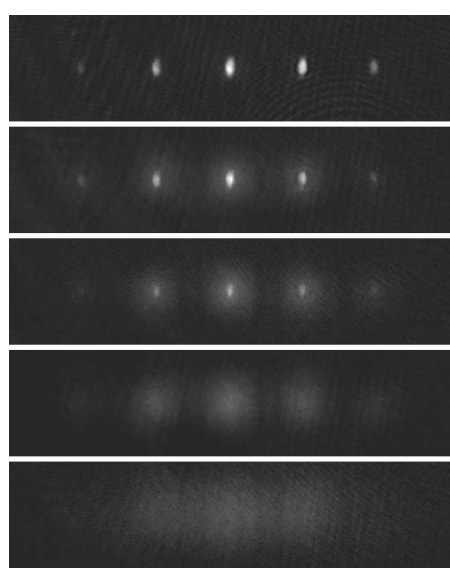

$\mathrm{T}(\mathrm{nK})$

$<200$

247

261

(d)

(e)

FIG. 5: Majorana transition with atoms at different temperature. From (a) to (e), the maximum optical density is 0.95 , $0.91,0.57,0.27$ and 0.23 , respectively.
In addition, the Majorana transition with atoms at different temperature is studied (see Fig. 5). The temperature of atoms is altered by setting the end frequency of evaporative cooling. As the temperature rises, the detected maximum optical density decreases, e.g., 0.95 in (a) yet 0.23 in (e). In fact, it is hard to get information of atoms in (e) because of the bad contrast. The lowest temperature of sub-Doppler cooling in $\mathrm{Rb}$ is $4 \mu \mathrm{K}$ [19] which is much higher than the temperature in (e). Even at about the critical temperature [see Fig. 5(d)], the small fractions of $m_{F}=2$ and $m_{F}=-2$ can hardly be detected. Another problem for atoms at high temperature is the overlapping of different component atoms [see Fig. 5(e)]. For these two reasons, BEC appeals to be the most suitable tool to study Majorana transition.

In conclusion, this work establishes a basic tool to control the macroscopic coherent quantum state of spinor condensates for developing more sophisticated control. As an application, multicomponent spinor atom laser is generated. The Majorana transition is precisely studied without the disturbance of atoms velocity distribution. Our treatment for Majorana transition leads to a quantitative agreement with the experimental results. With our model, the dynamical evolution of atoms prepared in any spinor state in variable magnetic field can be described. We also show the superiority of Bose-Einstein condensation as a tool to study Majorana transition.

\section{ACKNOWLEDGMENTS}

The authors thank Professor Jörg Schmiedmayer at Heidelberg University and Professor Li You at Georgia Institute of Technology for their helpful discussions. This work was supported by the National Fundamental Research Programme of China under Grant No. 2001CB309308, the Major Program of National Natural Science Foundation of China under Grant No. 60490280, National Natural Science Foundation of China under Grant No. 60271003 and 10474004.

* Electronic address: hongguo@pku.edu.cn

$\dagger$ Electronic address: xuzongchen@pku.edu.cn

[1] E. Majorana, Nuovo Cimento 9, 43 (1932).

[2] R. Frisch and E. Segrè, Z. Physik 80, 610 (1933).

[3] R. D. Hight and R. T. Robiscoe, Phys. Rev. A. 17, 561 (1978).

[4] J. E. Lye et al., J. Opt. B: Quantum Semiclassical Opt. 4, 57 (2002).

[5] H. Ott et al., Phys. Rev. Lett. 87, 230401 (2001).

[6] X. Ma et al., Phys. Rev. A. 73, 013624 (2006).

[7] S. Yi and H. Pu, Phys. Rev. Lett. 97, 020401 (2006).

[8] J. Kronjäger et al., Phys. Rev. Lett. 97, 110404 (2006).

[9] S. Mukerjee, C. Xu, and J. E. Moore, Phys. Rev. Lett. 97, 120406 (2006). 
[10] H. Saito, Y. Kawaguchi, and M. Ueda, Phys. Rev. Lett. 96, 065302 (2006).

[11] L. Santos, and T. Pfau, Phys. Rev. Lett. 96, 190404 (2006).

[12] R. B. Diener, and T. -L. Ho, Phys. Rev. Lett. 96, 190405 (2006).

[13] A. S $\phi$ rensen, L.-M. Duan, J. Cirac, and P. Zoller, Nature 409, 63 (2001).

[14] B. Julsgaard, A. Kozhekin, and E. Polzik, Nature 413,
400 (2001).

[15] H. Pu, and P. Meystre, Phys. Rev. Lett. 85, 3987 (2000).

[16] L. You and M. S. Chapman, Phys. Rev. A. 62, 052302 (2000).

[17] A. Meckler, Phys. Rev. 111, 1447 (1958).

[18] C. Zener, Proc. R. Soc. London, A137, 696 (1932).

[19] C. D. Wallace et al., J. Opt. Soc. Am. B 11, 703 (1994). 